\title{
Gain-Assisted Plasmon Resonance Narrowing and Its Application in Sensing
}

Meng, Lijun; Zhao, Ding; Yang, Yuanqing; García De Abajo, F. Javier; Li, Qiang; Ruan, Zhichao; Qiu, Min

Published in:

Physical Review Applied

Link to article, DOI:

10.1103/PhysRevApplied.11.044030

Publication date:

2019

Document Version

Publisher's PDF, also known as Version of record

Link back to DTU Orbit

Citation (APA):

Meng, L., Zhao, D., Yang, Y., García De Abajo, F. J., Li, Q., Ruan, Z., \& Qiu, M. (2019). Gain-Assisted Plasmon Resonance Narrowing and Its Application in Sensing. Physical Review Applied, 11(4), [044030].

https://doi.org/10.1103/PhysRevApplied.11.044030

\section{General rights}

Copyright and moral rights for the publications made accessible in the public portal are retained by the authors and/or other copyright owners and it is a condition of accessing publications that users recognise and abide by the legal requirements associated with these rights.

- Users may download and print one copy of any publication from the public portal for the purpose of private study or research.

- You may not further distribute the material or use it for any profit-making activity or commercial gain

- You may freely distribute the URL identifying the publication in the public portal 


\title{
Gain-Assisted Plasmon Resonance Narrowing and Its Application in Sensing
}

\author{
Lijun Meng, ${ }^{1,2,3}$ Ding Zhao, ${ }^{4}$ Yuanqing Yang, ${ }^{5}$ F. Javier García de Abajo, ${ }^{2,6}$ Qiang Li, ${ }^{3}$ \\ Zhichao Ruan, ${ }^{1,3, *}$ and Min Qiu ${ }^{3,7,8, \dagger}$ \\ ${ }^{1}$ Zhejiang Province Key Laboratory of Quantum Technology and Device, Department of Physics and State Key \\ Laboratory of Modern Optical Instrumentation, Zhejiang University, Hangzhou, Zhejiang, China \\ ${ }^{2}$ ICFO-Institut de Ciencies Fotoniques, The Barcelona Institute of Science and Technology, 08860 Castelldefels \\ (Barcelona), Spain \\ ${ }^{3}$ State Key Laboratory of Modern Optical Instrumentation, Zhejiang University, Hangzhou 310027, China \\ ${ }^{4}$ DTU Nanolab, Technical University of Denmark, 2800 Kongens Lyngby, Denmark \\ ${ }^{5}$ SDU Nano Optics, University of Southern Denmark, Campusvej 55, DK-5230 Odense, Denmark \\ ${ }^{6}$ ICREA-Institució Catalana de Recerca i Estudis Avançats, Passeig Lluís Companys 23, 08010 Barcelona, Spain \\ ${ }^{7}$ Institute of Advanced Technology, Westlake Institute for Advanced Study, 18 Shilongshan Road, \\ Hangzhou 310024, Zhejiang Province, China \\ ${ }^{8}$ Key Laboratory of $3 D$ Micro/Nano Fabrication and Characterization of Zhejiang Province, School of \\ Engineering, Westlake University, 18 Shilongshan Road, Hangzhou 310024, Zhejiang Province, China
}

(Received 31 July 2018; revised manuscript received 31 January 2019; published 10 April 2019)

We demonstrate the viability of using high-order modes in localized optical resonances combined with gain media to dramatically reduce the linewidths of absorption spectra. Our theoretical study provides a rational design route for small-footprint absorption with a high quality factor, which is typically a tradeoff for plasmonic light absorbers. Specifically, we design and numerically investigate a metal-insulatormetal absorber and a graphene Salisbury screen. Additionally, we study the potential application of these structures in sensing in order to achieve higher performance compared with conventional sensors based on the fundamental mode. Our approach, which can readily operate in a multiplexed fashion, has the potential for sensing minute amounts of analytes with a high figure of merit.

DOI: 10.1103/PhysRevApplied.11.044030

\section{INTRODUCTION}

Plasmonic nanostructures are routinely used to manipulate light at the nanoscale. Localized optical resonances constitute a basic ingredient in these structures. In particular, the collective oscillations of free electrons in conducting materials, known as surface plasmon resonances (SPRs), are able to interact strongly with external light and highly concentrate the electromagnetic intensity in the near field [1]. SPRs further facilitate energy conversion at the nanoscale, for example, through the generation of hot carriers $[2,3]$ and localized heating $[4,5]$. These appealing features have triggered intense research activities over the last two decades aimed at designing and exploiting different structures for nonlinear optics [6,7], ultrasensitive molecular detection via surface-enhanced Raman scattering (SERS) [8,9], photochemistry [10,11], and nanofabrication $[12,13]$, among other feats. Additionally, the design of light absorbers with high performance [14-19] is very

\footnotetext{
*zhichao@zju.edu.cn

†qiu_lab@westlake.edu.cn
}

appealing owing to their ability to strongly dissipate incoming light in any desirable spectral range. These optical elements are useful in several promising applications, including improved solar energy harvesters [20,21], novel photodetectors [22,23], refractive-index sensors [24,25], and selective thermal emitters according to Kirchhoff's law [26-29].

Absorbers are generally categorized into two types: broadband $[30,31]$ or narrowband $[15,16]$, depending on their absorption bandwidths. Reducing the bandwidth to an ultrasmall value is one of the longstanding goals in this field, which could enable higher-quality nanodevices for photodetection, sensing, and thermal emission. Various kinds of delocalized optical resonances are commonly used to realize ultranarrowband absorption [32-37] (e.g., lattice resonances [32-35] and guided-mode resonances [36,37]). This preference is partially due to their plasmonic-photonic hybrid-mode nature that features low dissipation rates. For localized resonances, in order to improve the quality factor $Q\left(=\omega_{0} / \Delta \omega\right.$, where $\omega_{0}$ is the peak frequency and $\Delta \omega$ is the full frequency width at half maximum), utilizing destructive interferences in complex structures to 
reduce radiative damping rates of the optical modes has been tried [38].

In this paper, we propose an alternative way consisting in utilizing high-order localized resonances combined with gain to realize high $Q$-factor perfect absorption within structures with small spatial extension. We consider a highorder optical mode that exhibits much smaller coupling strength with external light than the fundamental mode. Therefore, the energy stored in the high-order mode displays a small radiative decay rate. Simultaneously, gain partially compensates the intrinsic Ohmic losses in metal elements, leading to a small resistive decay rate. According to the critical-coupling condition from coupled-mode theory, perfect absorption with an ultrasmall bandwidth can be achieved when these two rates are equal.

In what follows, this concept is firstly demonstrated in metallodielectric core-shell cylinders, which support Mie resonances $[39,40]$. Then, we apply the idea to two popular planar absorbers: metal-insulator-metal $(M-I-M)$ structures and graphene Salisbury screens. They both validate the concept. Apart from their performance as light absorbers, the two structures also hold great potential for sensing $[15,41]$. This appealing feature is due to their highly confined near field and easy tunability of the resonance frequency through changing the geometrical size or doping level. Commonly, sensors based on localized resonances provide the opportunity to sense tiny volumes of analyte, even down to a nanometer-sized light focal spot [42]. But their spectra are broad, which limits the achievable improvement in the figure of merit (FOM) (see below). Based on our proposed small-footprint ultranarrowband absorbers, we further show that their performance for sensing exceeds that of sensors based on solely fundamental modes.

\section{ULTRANARROWBAND LIGHT DISSIPATION IN A NANOCYLINDER}

\section{A. Theory and methods}

Figure 1(a) shows the core-shell cylinders under consideration. The relative permittivity of the gold shell is approximated using a Drude model [43], $\varepsilon_{m}=1-$ $\omega_{p}^{2} /\left(\omega^{2}+i \omega \gamma_{p}\right)$, where $\hbar \omega_{p}=6.98 \mathrm{eV}, \gamma_{p}=0.11 \mathrm{eV}$. The core is a dielectric, which can be further doped with various gain materials. Then, the total electric response is $\varepsilon_{d}=\varepsilon_{b}+\varepsilon_{\text {gain }}$. It is composed of a background term $\varepsilon_{b}=2.25$ (i.e., silica) and a Lorentzian gain term [44,45] $\varepsilon_{\text {gain }}=F \gamma_{0} /\left(\omega-\omega_{0}+i \gamma_{0}\right)$. Here, $\omega_{0}$ and $\gamma_{0}$ are the gain frequency and bandwidth, respectively. In this section, we take Rhodamine 800 to provide the gain effect, whose emission wavelength $\lambda_{0}\left(=2 \pi c / \omega_{0}\right)$ is $711 \mathrm{~nm}$, and $\gamma_{0}=$ $0.04 \omega_{0}$ [45]. The explicit expression of $F$ shows that it is proportional to the population inversion of the gain material [45]. Therefore, it reflects the gain level and also includes a saturation effect. We emphasize that the required values of $F$ for our structures (see below) are smaller than its upper limit [45]. So, they are realistically attainable. Incidentally, we set $F=0$ when we want to exclude gain.

Modes with different orders (e.g., dipolar, quadrupolar mode, and other higher-order modes) can be excited by an (a)

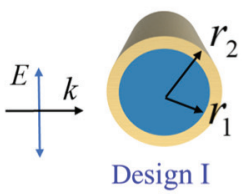

$r_{1}=34.9 \mathrm{~nm}, r_{2}=44.3 \mathrm{~nm}$

$\stackrel{y_{4}}{\longrightarrow z}$

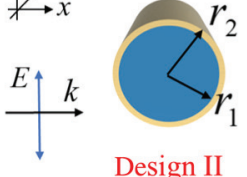

Design II

$r_{1}=40.2 \mathrm{~nm}, r_{2}=45.0 \mathrm{~nm}$ (b)

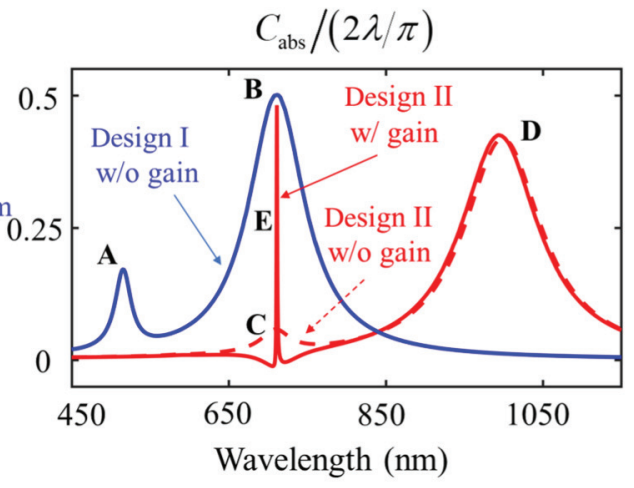

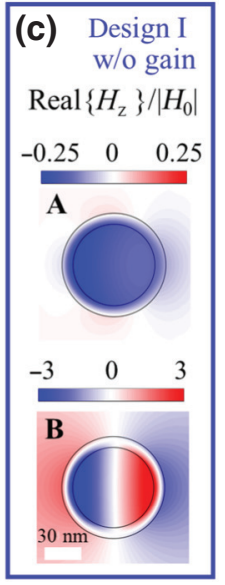

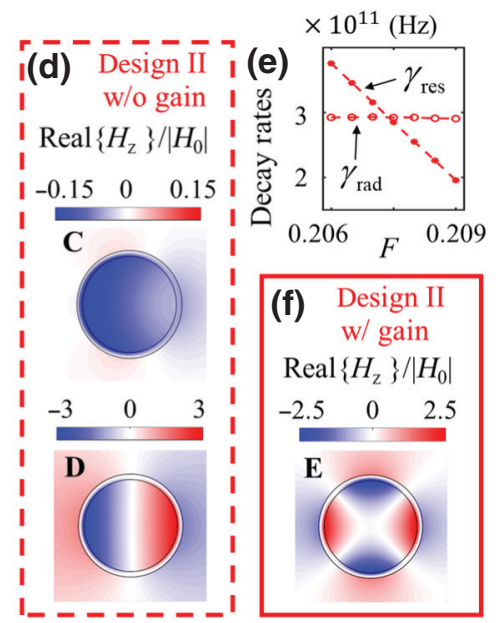

FIG. 1. (a) Schematic view of the two silica/gold core-shell structures here considered. (b) Normalized absorption cross sections of core-shell cylinders supporting a dipolar mode without gain (blue curve), a quadrupolar mode without gain (red dashed curve), or a quadrupolar mode with gain (red solid curve) at $711 \mathrm{~nm}$. (c) Normalized magnetic fields of a quadrupolar mode (peak A) and a dipolar mode (peak B), respectively, corresponding to the features labeled in the blue curve of (b). (d) Normalized magnetic fields of a quadrupolar mode (peak C) and a dipolar mode (peak D), respectively, associated with the red dashed curve in (b). (e) Radiative and resistive decay rates of a quadrupolar mode (related to peak E) at different gain levels. (f) Normalized magnetic field of the quadrupolar mode (peak E) of the red solid curve in (b) under the critical-coupling condition. Frames in (c),(d),(f) share the same color and line type as spectral curves in (b). Also, $\left|H_{0}\right|$ is the magnetic field amplitude of the incidence wave. 
external plane wave with its magnetic field polarized along the cylinder axis. Each mode is associated with a radiative decay rate $\left(\gamma_{\text {rad }}\right)$ and a resistive decay rate $\left(\gamma_{\text {res }}\right)$. They characterize the mode energy dissipation speeds either through coupling to the far field or generation of Joule heat in the metal. The absorption can reach a maximum value when the two decay rates are equal [46], a condition generally known as critical coupling. Specifically, for the cylinder discussed here, we have the absorption cross section of a single mode given by [47]

$$
C_{\mathrm{abs}}=2 \frac{2 \lambda}{\pi} \frac{\gamma_{\mathrm{rad}} \gamma_{\mathrm{res}}}{\left(\omega-\omega_{0}\right)^{2}+\left(\gamma_{\mathrm{rad}}+\gamma_{\mathrm{res}}\right)^{2}},
$$

where the prefactor of 2 accounts for two degenerate submodes because of the structure's rotational symmetry. One can find the upper limit to be $C_{\mathrm{abs}, \max }=(2 \lambda / \pi) / 2$, which can be achieved when both critical-coupling and on-resonance conditions are satisfied.

In the numerical experiments that we discuss next, we use the eigenfrequency solver of the commercial software COMSOL [48] to compute the two decay rates. The method is discussed in Appendix A. Because the cylinder as well as the other two studied structures (see below) are all invariant under translations along one direction and we consider the magnetic fields of the incidence light to be parallel with the direction, the resulting magnetic field associated with the near field also satisfies this condition. In the computation, a circular simulation region is used, defined by a scattering boundary. The structure (i.e., a core-shell circle) is located at the center. The distance between the structure and the scattering boundary is set to be larger than the light wavelength. In the simulations of absorption spectra, plane waves at different wavelengths going toward the positive $x$ direction are set as the background fields in the frequency domain solver. Then, scattered fields are computed. In the two-dimensional (2D) structure, the absorption cross section in units of meters is equal to the absorbed energy of the total fields (units of $\mathrm{W} / \mathrm{m}$ ) divided by the incidence intensity (units of $\mathrm{W} / \mathrm{m}^{2}$ ).

\section{B. Results and discussion}

In many applications, the fundamental dipolar mode is typically preferred and no gain medium is involved. Because the mode has large coupling strength with the external light, it is therefore easily accessible. Design I, presented in Fig. 1(a), illustrates such a case. As indicated by peak B of the blue curve in Fig. 1(b), its maximum absorption is achieved at $711 \mathrm{~nm}$. The corresponding normalized scattered magnetic field is shown in panel B of Fig. 1(c). The geometric parameters of the core-shell cylinder are $r_{1}=34.9 \mathrm{~nm}$ and $r_{2}=44.3 \mathrm{~nm}$. Obviously, the incidence light can excite higher-order modes at shorter wavelengths. For example, the small peak $A$ at $516 \mathrm{~nm}$ reveals a quadrupolar mode. Panel A of Fig. 1(c) displays the corresponding field distribution, which has a characteristic four-lobed pattern, but is far from being a well-defined quadrupolar mode [see Fig. 1(f), discussed below]. The reason lies in the large mismatch between the two energy decay rates. Although the peak absorbance induced by the dipolar mode can be high, the bandwidth is large with a full wavelength width at half maximum FWHM $=85 \mathrm{~nm}$, leading to a low $Q$ factor (approximately 8). This results from the large radiative and resistive decay rates associated with a typical fundamental mode.

Adopting gain-assisted higher-order modes can address this problem. For the sake of a fair comparison, we use the eigenfrequency solver to find a new core-shell cylinder design II, which holds a quadrupolar mode at the same wavelength (i.e., $711 \mathrm{~nm}$ ) as that of the dipolar mode of design I. The new structure's geometric parameters are $r_{1}=40.2 \mathrm{~nm}$ and $r_{2}=45.0 \mathrm{~nm}$. The red dashed curve in Fig. 1(b) shows its normalized absorption cross section. Peaks C and D correspond to its quadrupolar mode and dipolar mode, respectively. Their field distributions are shown in Fig. 1(d). Then, we add gain (Rhodamine 800, discussed above) into the silica core. Figure 1(e) depicts the dependences of the two decay rates of the quadrupolar mode on gain strength $F$. Within the explored range, with increasing optical gain, the values of the radative decay rates $\gamma_{\text {rad }}$ remain almost the same, while the resistive decay rates $\gamma_{\text {rad }}$ become increasingly smaller. The criticalcoupling condition is achieved around $F=0.2075$. The red solid curve in Fig. 1(b) is the normalized absorption cross section under this condition. We can see from the peak $\mathrm{E}$ that nearly perfect absorption $\left[C_{\mathrm{abs}} /(2 \lambda / \pi)=\right.$ $0.48]$ is produced at the target wavelength. Furthermore, the bandwidth is dramatically reduced to a value as small as $1 \mathrm{~nm}$. The reason why the peak absorption is slightly below the upper limit (i.e., 0.5) is that, here, absorption is dominant, but does not fully originate in the quadrupolar mode. It is also influenced by the nearby dipolar mode. The field distribution related to peak $\mathrm{E}$ is shown in Fig. 1(f), which confirms an efficient excitation of the quadrupole mode assisted by gain.

The imaginary part of the relative permittivity of the doped dielectric can be written as $\operatorname{Im}\left\{\varepsilon_{\text {gain }}\right\}=$ $-F /\left[\left(\Delta \omega / \gamma_{0}\right)^{2}+1\right]$, where $\Delta \omega=\omega-\omega_{0}$. We can find that a small gain bandwidth $\gamma_{0}$ can also contribute to a reduction of the linewidths of the absorption spectra. In order to clarify its role in our structure, we additionally show in Fig. 2 the absorption spectra with $\gamma_{0}$ taking different values. The red curve in Fig. 2 is the same as the red solid one (peak E) in Fig. 1(b). Although one can observe that the absorption bandwidth increases with increasing gain bandwidth, it remains as small as approximately 1.5 $\mathrm{nm}$ when $\gamma_{0}$ takes a considerably large value of $0.4 \omega_{0}$. This reveals that the ultranarrowband is a unique advantage, mainly resulting from the combination of a high-order mode and gain, rather than from the small gain bandwidth. 


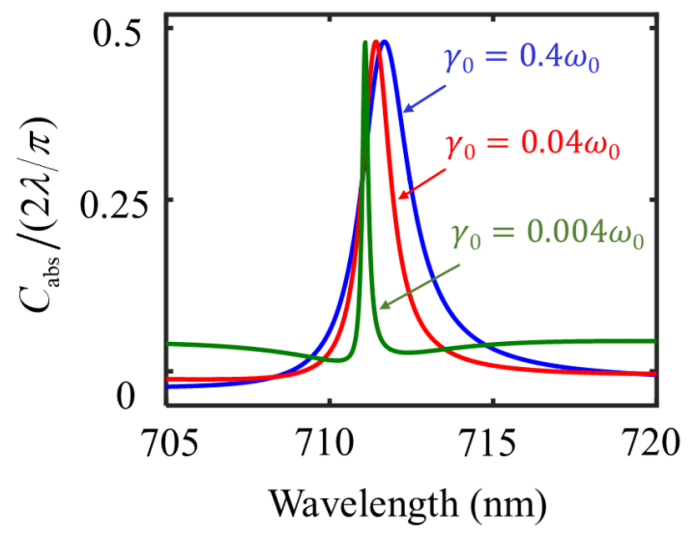

FIG. 2. Normalized absorption cross sections related to the peak E in Fig. 1(a) when the gain bandwidth of the gain medium takes different values.

Incidentally, we also note that a single structure is only able to dissipate a small fraction of energy of an incidence plane wave or Gaussian beam. As we discuss below, arranging several such structures together can address this problem.

\section{METAL-INSULATOR-METAL ABSORBER}

\section{A. Theory and methods}

Now, we apply the same idea to solid-state planar absorbers, which are usually made up of periodic arrays of subwavelength structures. For this type of absorber, coupled-mode theory has also been used as a wellestablished formalism for the analysis of the absorbance by using the two decay rates [36,49]: $A=4 \gamma_{\mathrm{rad}} \gamma_{\mathrm{res}} /[(\omega-$ $\left.\left.\omega_{0}\right)^{2}+\left(\gamma_{\text {rad }}+\gamma_{\text {res }}\right)^{2}\right]$. Again, we can see that the maximum absorbance can be achieved on resonance under the critical-coupling condition. In this section, we focus on the popular $M-I-M$ absorbers. The inset of Fig. 3(a) shows a single unit. A dielectric spacer $\left(\varepsilon_{b}=1.75^{2}\right.$, i.e., $\mathrm{Al}_{2} \mathrm{O}_{3}$ ) with thickness $t$ is sandwiched between a continuous gold film and a gold strip. The width and the height of the strip are $w$ and $d$, respectively. Also, $a$ denotes the period. The upper and lower metal elements can interact strongly if the dielectric layer is thin enough. When carefully designed, the impinging plane wave with magnetic field along the strip can induce antiparallel electrical currents in the two metal elements, therefore generating a remarkable magnetic resonance in the gap $[15,16]$. When considering gain, this section adopts the same gain medium (i.e., Rhodamine 800 ) as that in the last section.

Here, we study both infinite and finite arrays of $M-I-M$ absorbers. The performance of infinitely large structures is presented in Figs. 3(a)-3(c), where we compare structures supporting only a fundamental mode without gain to others supporting a higher-order mode with gain. In the simulations, periodic boundary conditions in the $x$ direction and perfect matching layers (PMLs) in the $y$ direction are adopted. A port below the upper PML illuminates a plane wave toward the structure. The distance between the port and the structure is set to be larger than the light wavelength. The performance of finite arrays for light absorption and sensing is displayed in Figs. 3(d) and 4, respectively. In the simulation of Fig. 3(d), a substrate (an opaque metal film plus a thin dielectric layer) with a width of $6 \mu \mathrm{m}$ is assumed to be freely standing in air. A finite number of metal ribbons sit on the substrate. PMLs are assumed in all four directions. The distances between the PMLs and the structure are set to be larger than the light wavelengths. A 2D Gaussian beam with its waist equal to $711 \mathrm{~nm}$ (i.e., the resonant wavelength) is taken to (a)

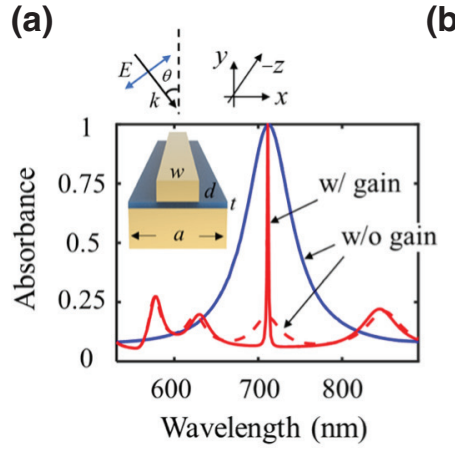

(b)

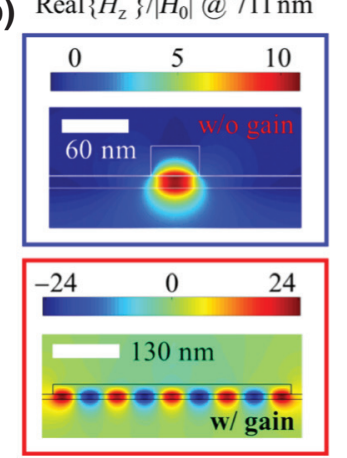

(c)

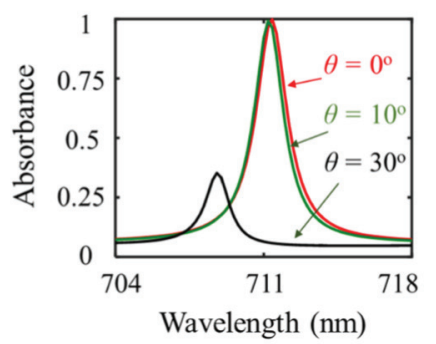

(d)

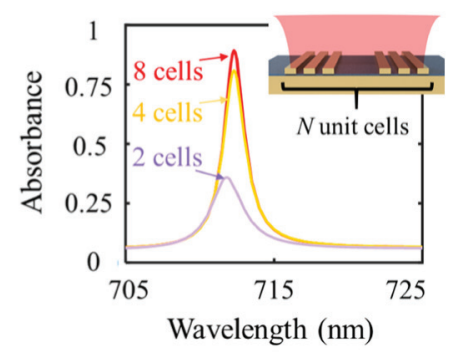

FIG. 3. Absorption properties of infinite one-dimensional (1D) arrays illuminated by plane waves (a)-(c) and finite arrays illuminated by Gaussian beams (d). (a) Absorption spectra of the absorbers, respectively, supporting a fundamental mode without gain (blue curve), a higher-order mode without gain (red dashed curve), or a higher-order mode with gain (red solid curve) at $711 \mathrm{~nm}$. The inset depicts the structure. (b) On-resonance normalized magnetic fields under the two different conditions considered in (a). The frames share the same color and line type as spectral curves in (a). (c) Absorption spectra at different incidence angles. (d) Absorption spectra for finite arrays of different size (i.e., number of unit cells). Both (c) and (d) correspond to the higher-order-mode structures. 
(a)
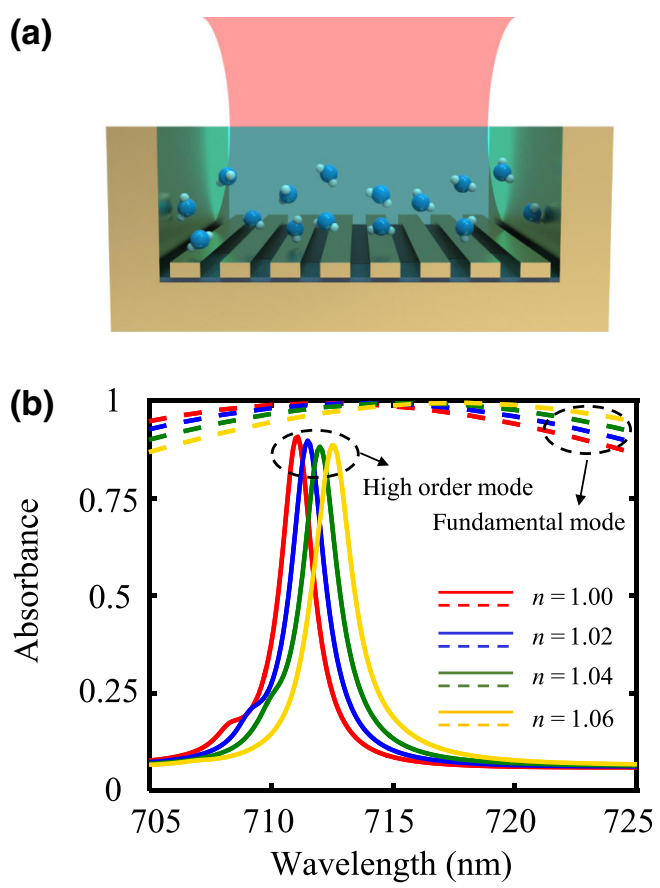

FIG. 4. (a) A schematic view of a nanodevice for gas sensing. Grooves are filled with the targeted gas of index $n$. (b) Absorption spectra when the gas takes different indices. The solid (dashed) curves correspond to the structure utilizing a higher-order (fundamental) mode.

normally illuminate the structure. The waist is at the interface between the dielectric spacer and the metal ribbons. The whole system is symmetric with respect to the central vertical line.

In the simulation of Fig. 4, the same Gaussian beam is set as the excitation source with its waist at the interface between the gold groove and the air. The detected gas with refractive index $n$ is assumed to fill the groove. The thickness of the wall of the groove is larger than the light wavelength. The three sides (left, right, and bottom) of the groove are enclosed by gold PMLs. The three sides (left, right, and top) of the air are also enclosed by air PMLs. The size of the groove itself is discussed below.

\section{B. Results and discussion}

For $M-I-M$ absorbers, researchers normally use a fundamental mode as displayed by the field profile in the upper panel of Fig. 3(b). Its absorption spectrum is indicated by the blue curve in Fig. 3(a) with complete absorption at $711 \mathrm{~nm}$. The geometric parameters are $w=48 \mathrm{~nm}$, $d=30 \mathrm{~nm}, t=12 \mathrm{~nm}$, and $a=250 \mathrm{~nm}$. We note that the large FWHM (approximately $70 \mathrm{~nm}$ ) leads to a fairly low $Q$ factor (approximately 10). In order to achieve high$Q$ perfect absorption, we follow a method similar to that shown in the last section to adopt a new $M-I-M$ structure, which holds a higher-order resonance at the target wavelength (i.e., $711 \mathrm{~nm}$ ). The dielectric spacer is doped with the gain medium. When the geometric parameters take values of $w=476 \mathrm{~nm}, d=20 \mathrm{~nm}, t=10 \mathrm{~nm}$, and $a=$ $520 \mathrm{~nm}$ and the gain strength $F$ is tuned to 0.171 , complete absorption with FWHM $=2 \mathrm{~nm}$ is achieved [red solid curve in Fig. 3(a)]. The $Q$ factor is enhanced up to approximately 356 . The on-resonance field distribution is displayed in the lower panel of Fig. 3(b). If no gain medium is involved (i.e., $F=0$ ), the peak absorbance dramatically drops to about 0.2 as shown by the red dashed curve in Fig. 3(a). Figure 3(c) shows the angle-dependence absorption of the higher-order-mode structure with gain. The resonance blueshifts and becomes much weaker when the incidence angle is increased to $30^{\circ}$, which is due to the spatially large optical resonance mode compared with the light wavelength.

We have so far investigated absorbers with an infinite period, in line with many previously published numerical studies. Now, based on this kind of high- $Q$ localized resonance, we can realize small-footprint ultranarrowband absorbers, which is demonstrated in Fig. 3(d). All geometric parameters (of the ribbon and the period) and material parameters are the same as those of the highorder-mode infinite arrays. We can observe that the peak absorbance grows remarkably when the unit cell number increases from 2 to 8 . Specifically, as indicated by the yellow curve, a small-area absorber, which consists of 4 unit cells (about 1.5 times the beam waist), can dissipate $80 \%$ of the incoming light energy with a bandwidth less than $3 \mathrm{~nm}$.

These properties are useful for sensing small volume analytes with a higher FOM compared with using the fundamental resonance. Figure 4(a) presents a sketch of the designed sensor. The absorber, which consists of 8 unit cells, is integrated onto the bottom of a gold groove. This means that the width of the groove is $4.16 \mu \mathrm{m}$. The depth of the groove is assumed to be $1 \mu \mathrm{m}$. The solid curves in Fig. 4(b) illustrate the dependence of the absorption spectra on the gas refractive index (see figure legend). For comparison, we also do calculations of a sensor that utilizes the absorber based on the fundamental mode as described in Figs. 3(a) and 3(b) without changing the other parameters (i.e., the depth and width of the groove). This means that there are 16 unit cells in the absorber. The results are indicated by the dashed curves. We find that while the sensitivity $(S=\Delta \lambda / \Delta n)$, which measures the variation of peak wavelengths per refractive index unit (RIU), of the higher-order-mode structure ( $25 \mathrm{~nm} / \mathrm{RIU})$ is smaller than the one based on the fundamental mode $(83 \mathrm{~nm} / \mathrm{RIU})$, its FOM $=S / \mathrm{FWHM}$ is one order of magnitude larger. The FOMs of the higher-order-mode and the fundamental-mode structures are 8.3/RIU and 0.8/RIU, respectively. 


\section{GRAPHENE SALISBURY SCREEN}

\section{A. Theory and methods}

Apart from the visible range, absorbers working in the MIR region are of great interest as well, especially for applications in sensing of molecular vibrational bonds and thermal imaging [50]. In this context, graphene plasmons have raised huge expectations owing to their outstanding optical properties in this spectral range [51,52]. Perfect absorption has been demonstrated both theoretically [53] and experimentally [54]. Here, we shift our focus from the conventionally used dipolar resonance to higher-order resonances, showing that narrower linewidths are possible. A unit cell of the structure is shown in the inset of Fig. 5(a). A thick dielectric layer with thickness $t\left(\varepsilon_{b}=2\right.$, i.e., $\left.\mathrm{GaF}_{2}\right)$ separates a graphene nanoribbon and a gold film. The width of the ribbon is $w$ and the period is $a$. The surface conductivity of the graphene layer is described by a Drude model $\sigma=\left(e^{2} / \pi \hbar^{2}\right) i E_{F}\left(\omega+\mathrm{i} \tau^{-1}\right)$, where $\tau=\mu E_{F} / e v_{F}^{2}$ is the intrinsic inelastic lifetime. In the simulation, we assume $E_{F}=0.8 \mathrm{eV}, \mu=2.5 \times 10^{4} \mathrm{~cm}^{2} \mathrm{~V}^{-1} \mathrm{~s}^{-1}$, and $v_{F}=10^{6} \mathrm{~m} \mathrm{~s}^{-1}$. Here, we take $\mathrm{Er}^{3+}$ ions as a gain medium when the gain is needed, whose emission wavelength is $\lambda_{0}=2800 \mathrm{~nm}$, and the gain bandwidth is $\gamma_{0}=0.03 \omega_{0}[45]$.

In what follows, we carry out an analysis along similar lines as in the previous section. Firstly, we focus on infinitely large structures that support either a fundamental dipolar mode or a higher-order mode at the same wavelength $(2800 \mathrm{~nm})$. The results are presented in Figs. 5(a)-5(c). Then, we also investigate finite arrays for small-footprint high- $Q$ absorption [Fig. 5(d)] and sensing (Fig. 6), respectively. The setups that we consider in our simulations, including boundary conditions, beam types, and domain sizes, are almost the same as those in the previous section. The difference is that the waists of the Gaussian beams in Figs. 5(d) and 6 are equal to $2800 \mathrm{~nm}$. Correspondingly, the width of the substrates is extended to $12 \mu \mathrm{m}$ to support the finite arrays and totally block transmission of the incident light. In the simulations of sensing, as indicated in Fig. 6(a), the graphene ribbon array is covere by a 10 -nm-thick molecular layer, whose width is $3.5 \mu \mathrm{m}$ (see below). The molecular layer is simply described as a refractive index that increases with increasing molecular density [55].

\section{B. Results and discussion}

In the design of the screen, we set the filling fraction of the graphene ribbon to be 0.5 (i.e., $w=a / 2$ ). When $w, a$, and $t$ take values of 16,32 , and $885 \mathrm{~nm}$, respectively, the ribbon holds a dipolar resonance at $2800 \mathrm{~nm}$ under normal incidence. The field distribution is shown in the upper panel of Fig. 5(b). Its absorption spectrum [blue curve in Fig. 5(a)] illustrates that perfect absorption is achieved with FWHM $=5 \mathrm{~nm}$. Next, we reconfigure the absorber with $w=214 \mathrm{~nm}, a=428 \mathrm{~nm}$, and $t=481 \mathrm{~nm}$, so that it holds a higher-order resonance at the same wavelength. The critical-coupling condition can be realized, when a proper strength of gain $(F=0.0018)$ is added into the dielectric layer to partially compensate the intrinsic Ohmic loss of the system. Then, as indicated by the red solid curve in Fig. 5(a), perfect absorption is achieved with a narrower linewidth FWHM $=0.8 \mathrm{~nm}$. The lower panel of Fig. 5(b) displays its on-resonance field distribution. If there is no gain involved, the peak absorbance drops to around 0.5 (red dashed curve). Figure 5(c) presents the dependence of the absorbance on wavelength and incidence angle. One can observe that the ultranarrowband feature is robust over
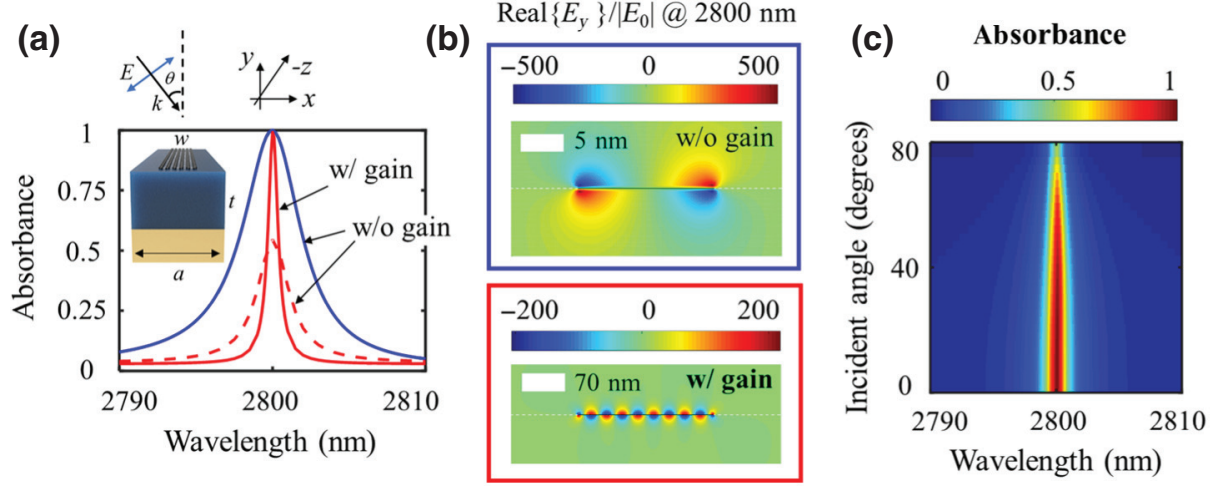

(d) Small footprint absorber

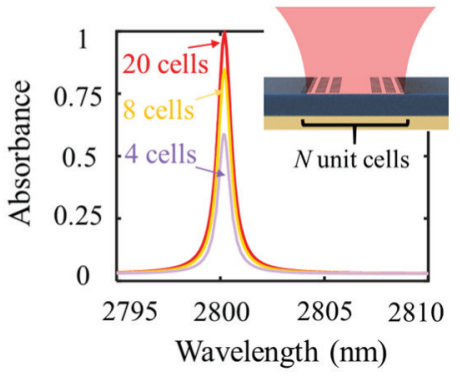

FIG. 5. Absorption properties of infinite 1D arrays illuminated by plane waves (a)-(c) and finite arrays illuminated by Gaussian beams (d). (a) Absorption spectra of absorbers supporting a fundamental mode without gain (blue curve), a higher-order mode without gain (red dashed curve), or a higher-order mode with gain (red solid curve) at $2800 \mathrm{~nm}$. The inset depicts the structure under consideration. (b) On-resonance normalized magnetic fields under the two different conditions examined in (a). The frames share the same color and line type as spectral curves in (a). (c) Absorbance as a function of incidence angle and wavelength. (d) Absorption spectra for finite arrays of different size (i.e., number of unit cells). Both (c) and (d) correspond to the higher-order-mode structures. 
a large angular range. The absorbance remains about 0.5 when the angle is increased to $80^{\circ}$.

In the same way as for the configuration in Fig. 3(d) discussed in the previous section, Fig. 5(d) shows the absorption performance of a finite screen. All parameters are the same as those of the infinite structure that holds a high-order mode. Thanks to the large optical cross section and robust angle of the graphene ribbon, 20 unit cells, which span about 1.53 times the beam waist, are enough to fully dissipate the incoming light; meanwhile, the bandwidth remains close to $1 \mathrm{~nm}$.

The ultraconfinement of the near field provided by the graphene plasmons renders this material particularly promising for molecular sensing [41]. Based on the smallfootprint high- $Q$ absorber, we have the capability to sense tiny volumes of the sample with improved FOM compared to its counterpart relying on the fundamental resonance. The sensor, which supports the high-order resonance and involves gain, consists of 8 unit cells. The molecular layer with width $3.5 \mu \mathrm{m}$ fully covers these cells. For comparison, we replace the high-order resonance structure with one that holds the fundamental resonance without gain. At the same time, we maintain the widths of the ribbon array and the molecular layer to be the same, which means that the fundamental-mode structure contains 107 unit cells. Figure 6(b) displays their absorption spectra with various
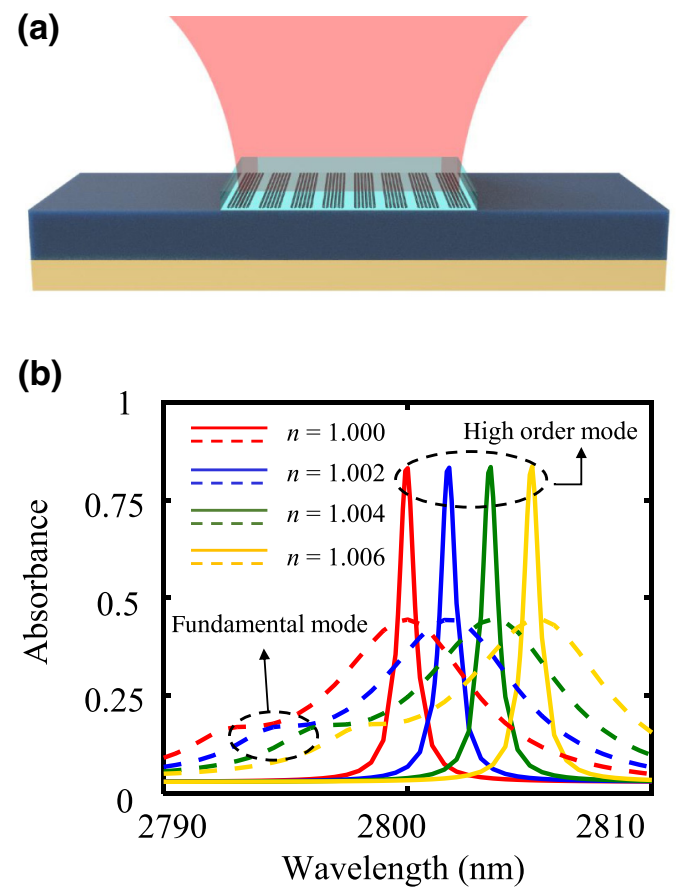

FIG. 6. (a) Schematic view of a nanodevice for thin molecular layer sensing. The molecular layer with a thickness of $10 \mathrm{~nm}$ is characterized by a refractive index $n$. (b) Absorption spectra for different molecular layer indices. The solid (dashed) curves correspond to the structure utilizing the higher-order (fundamental) resonance. layer indices tuned from 1 to 1.006. Although their sensitivities are almost the same, the high-order-resonancebased sensor holds a larger FOM (944/RIU) compared with its fundamental-mode counterpart, whose FOM is 131/RIU.

We note that the absorption of the finite structure supporting the fundamental resonance is fairly low (see dashed curves) compared with its infinite counterpart [blue curve in Fig. 5(a)]. We attribute this effect to the small distances between adjacent ribbons $(a-w=16 \mathrm{~nm})$, which leads to a strong near-field coupling between them. As a consequence, the optical mode is partially of lattice resonance in nature. Therefore, in this case, a much larger finite structure is necessary to recover the performance of the infinite array.

\section{CONCLUSION}

In summary, we have provided a general route to reduce the bandwidth while maintaining perfect absorption in a plasmonic system. We have realized this through using high-order optical resonances combined with proper gain, pumped by either light or electrical means. We demonstrate this strategy through numerical simulations of three different structures based on different absorption mechanisms. We further exploit these results to achieve improved performance in refractive-index sensing.

Incidentally, even better performances can be expected if much higher-order resonances are used. We hope that the present work stimulates further investigations for applying our method to customize absorption in other plasmonic systems. Finally, we expect that this idea can also benefit ultrahigh-quality-factor absorber/emitter designs in dielectric nanophotonics [56], where a gain medium is perhaps not needed because of the low intrinsic loss. Nevertheless, plasmonic systems have their unique advantages for some applications such as hot-electron generation with smallfootprint structures, as well as switchable photodetectors.

\section{ACKNOWLEDGMENTS}

This work has been supported in part by National Key Research and Development Program of China (Grants No. 2017YFA0205700 and No. 2017YFE0100200); National Natural Science Foundation of China (NSFC) (Grants No. 61425023 , No. 61575177, 61775194, No. 61675179, and No. 91850108); and Spanish MINECO Grant No. PCIN2015-155. D.Z. is funded from the European Union's Horizon 2020 research and innovation program under the Marie Curie grant (Grant No. 713683). F.J.G.d.A. acknowledges ERC (Advanced Grant No. 789104-eNANO), the Spanish MINECO (Grants No. MAT2017-88492-R and No. SEV2015-0522), AGAUR (Grant No. 2014 SGR 1400), and Fundació Privada Cellex. 


\section{APPENDIX: NUMERICAL METHOD USED TO COMPUTE RESISTIVE AND RADIATIVE DECAY RATES}

We describe in detail the method used to obtain Fig. 1(e). The structure in the corresponding COMSOL simulation is described at the end of Secs. II A. For a specific coreshell structure, we obtain the frequency of a desired optical eigenmode via the eigenfrequency solver. The solved eigenfrequency $\hat{\omega}=\omega_{0}+\gamma_{\text {tot }}$ is a complex number, where $\omega_{0}$ denotes the angular frequency of the eigenmode, while $\gamma_{\text {tot }}=\gamma_{\text {res }}+\gamma_{\text {rad }}$ is the sum of two decay rates: resistive and radiative. The exact values of these two rates can be calculated as [57] $\gamma_{\text {res }}=\gamma_{\text {tot }} \times W_{\text {res }} /\left(W_{\text {res }}+W_{\text {rad }}\right)$ and $\gamma_{\text {rad }}=\gamma_{\text {tot }} \times W_{\text {rad }} /\left(W_{\text {res }}+W_{\text {rad }}\right)$. The internally dissipated power $W_{\text {res }}$, which is equal to the Ohmic loss partially compensated by optical gain, can be obtained by $2 \mathrm{D}$ integration of the absorbed power density $P=(1 / 2) \varepsilon_{0} \omega_{0} \operatorname{Im}\left\{\varepsilon_{r}\right\}|E|^{2}$ over the whole structure. Here, $\varepsilon_{0}$ is the vacuum permittivity and $\varepsilon_{r}$ is the relative permittivity of either the lossy metal or the doped dielectric. The out-flux power $W_{\text {rad }}$ can be readily obtained by using a built-in function, which performs 1D integration of the normal component of timeaveraged Poynting vector $\langle\mathbf{S}\rangle=\langle\mathbf{E} \times \mathbf{H}\rangle$ along a closed contour enclosing the structure.

Apart from isolated structures, this method can also be applied to infinitely extended ones (e.g., the $M-I-M$ absorber and the graphene Salisbury screen, although we omit the results for simplicity). The difference with respect to finite structures is that now the contour for 1D integration of the Poynting vector can be taken as a straight line, which is placed above the structure and connects the two periodic boundaries in a single unit cell modeling. Then, the integral collects all the radiatively emitted power going to the upper space. Incidentally, for a graphene ribbon, the otherwise zero-thickness interface, which is specified by the surface conductivity $\sigma$, can be replaced by a finitethickness layer described by its relative permittivity $\varepsilon_{r}$. The formula $\varepsilon_{r}=1+\mathrm{i} \sigma /\left(\omega t \varepsilon_{0}\right)$ [58] is used to connect the two quantities, where the graphene ribbon thickness $t$ is adjusted in order to make the optical response (e.g., the absorption cross section) based on the two methods identical. Then, the 2D integration of the absorbed power density can be carried out as usual.

[1] S. A. Maier, Plasmonics: Fundamentals and Applications (Springer Science \& Business Media, 2007).

[2] R. Sundararaman, P. Narang, A. S. Jermyn, W. A. Goddard III, and H. A. Atwater, Theoretical predictions for hot-carrier generation from surface plasmon decay, Nat. Commun. 5, 5788 (2014).

[3] M. L. Brongersma, N. J. Halas, and P. Nordlander, Plasmon-induced hot carrier science and technology, Nat. Nanotechnol. 10, 25 (2015).
[4] G. Baffou and R. Quidant, Thermo-plasmonics: Using metallic nanostructures as nano-sources of heat, Laser. Photon. Rev. 7, 171 (2013).

[5] L. Meng, R. Yu, M. Qiu, and F. J. García de Abajo, Plasmonic nano-oven by concatenation of multishell photothermal enhancement, ACS Nano 11, 7915 (2017).

[6] M. Danckwerts and L. Novotny, Optical Frequency Mixing at Coupled Gold Nanoparticles, Phys. Rev. Lett. 98, 026104 (2007).

[7] S. Kim, J. Jin, Y.-J. Kim, I.-Y. Park, Y. Kim, and S.-W. Kim, High-harmonic generation by resonant plasmon field enhancement, Nature 453, 757 (2008).

[8] K. Kneipp, Y. Wang, H. Kneipp, L. T. Perelman, I. Itzkan, R. R. Dasari, and M. S. Feld, Single Molecule Detection using Surface-enhanced Raman Scattering (sers), Phys. Rev. Lett. 78, 1667 (1997).

[9] S. Nie and S. R. Emory, Probing single molecules and single nanoparticles by surface-enhanced Raman scattering, Science 275, 1102 (1997).

[10] C. Chen and R. Osgood, Direct Observation of the Localfield-enhanced Surface Photochemical Reactions, Phys. Rev. Lett. 50, 1705 (1983).

[11] S. Mukherjee, F. Libisch, N. Large, O. Neumann, L. V. Brown, J. Cheng, J. B. Lassiter, E. A. Carter, P. Nordlander, and N. J. Halas, Hot electrons do the impossible: Plasmon-induced dissociation of $\mathrm{H} 2$ on $\mathrm{Au}$, Nano Lett. 13, 240 (2012).

[12] E. C. Garnett, W. Cai, J. J. Cha, F. Mahmood, S. T. Connor, M. G. Christoforo, Y. Cui, M. D. McGehee, and M. L. Brongersma, Self-limited plasmonic welding of silver nanowire junctions, Nat. Mater. 11, 241 (2012).

[13] P. Ghosh, J. Lu, Z. Chen, H. Yang, M. Qiu, and Q. Li, Photothermal-induced nanowelding of metal-semiconductor heterojunction in integrated nanowire units, Adv. Electron. Mater. 4, 1700614 (2018).

[14] X. Liu, T. Starr, A. F. Starr, and W. J. Padilla, Infrared Spatial and Frequency Selective Metamaterial with Near-unity Absorbance, Phys. Rev. Lett. 104, 207403 (2010).

[15] N. Liu, M. Mesch, T. Weiss, M. Hentschel, and H. Giessen, Infrared perfect absorber and its application as plasmonic sensor, Nano Lett. 10, 2342 (2010).

[16] J. Hao, J. Wang, X. Liu, W. J. Padilla, L. Zhou, and M. Qiu, High performance optical absorber based on a plasmonic metamaterial, Appl. Phys. Lett. 96, 251104 (2010).

[17] L. Meng, D. Zhao, Q. Li, and M. Qiu, Polarizationsensitive perfect absorbers at near-infrared wavelengths, Opt. Express 21, A111 (2013).

[18] A. Alu and N. Engheta, Input Impedance, Nanocircuit Loading, and Radiation Tuning of Optical Nanoantennas, Phys. Rev. Lett. 101, 043901 (2008).

[19] D. Zhao, L. Meng, H. Gong, X. Chen, Y. Chen, M. Yan, Q. Li, and M. Qiu, Ultra-narrow-band light dissipation by a stack of lamellar silver and alumina, Appl. Phys. Lett. 104, 221107 (2014).

[20] H. A. Atwater and A. Polman, Plasmonics for improved photovoltaic devices, Nat. Mater. 9, 205 (2010).

[21] O. Neumann, A. S. Urban, J. Day, S. Lal, P. Nordlander, and N. J. Halas, Solar vapor generation enabled by nanoparticles, ACS Nano 7, 42 (2012). 
[22] M. W. Knight, H. Sobhani, P. Nordlander, and N. J. Halas, Photodetection with active optical antennas, Science 332, 702 (2011).

[23] H. Chalabi, D. Schoen, and M. L. Brongersma, Hotelectron photodetection with a plasmonic nanostripe antenna, Nano Lett. 14, 1374 (2014).

[24] M. E. Stewart, C. R. Anderton, L. B. Thompson, J. Maria, S. K. Gray, J. A. Rogers, and R. G. Nuzzo, Nanostructured plasmonic sensors, Chem. Rev. 108, 494 (2008).

[25] J. Anker, W. Hall, O. Lyandres, N. Shah, J. Zhao, and R. Van Duyne, Biosensing with plasmonic nanosensors, Nat. Mater. 7, 442 (2008).

[26] J.-J. Greffet, R. Carminati, K. Joulain, J.-P. Mulet, S. Mainguy, and Y. Chen, Coherent emission of light by thermal sources, Nature 416, 61 (2002).

[27] X. Liu, T. Tyler, T. Starr, A. F. Starr, N. M. Jokerst, and W. J. Padilla, Taming the Blackbody with Infrared Metamaterials as Selective Thermal Emitters, Phys. Rev. Lett. 107, 045901 (2011).

[28] K.-K. Du, Q. Li, Y.-B. Lyu, J.-C. Ding, Y. Lu, Z.-Y. Cheng, and M. Qiu, Control over emissivity of zero-static-power thermal emitters based on phase-changing material GST, Light: Sci. Appl. 6, e16194 (2017).

[29] Y. Qu, Q. Li, L. Cai, M. Pan, P. Ghosh, K. Du, and M. Qiu, Thermal camouflage based on the phase-changing material GST, Light: Sci. Appl. 7, 26 (2018).

[30] K. Aydin, V. E. Ferry, R. M. Briggs, and H. A. Atwater, Broadband polarization-independent resonant light absorption using ultrathin plasmonic super absorbers, Nat. Commun. 2, 517 (2011).

[31] W. Wang, Y. Qu, K. Du, S. Bai, J. Tian, M. Pan, H. Ye, M. Qiu, and Q. Li, Broadband optical absorption based on single-sized metal-dielectric-metal plasmonic nanostructures with high- $\varepsilon^{\prime \prime}$ metals, Appl. Phys. Lett. 110, 101101 (2017).

[32] S. R. K. Rodriguez, A. Abass, B. Maes, O. T. Janssen, G. Vecchi, and J. G. Rivas, Coupling Bright and Dark Plasmonic Lattice Resonances, Phys. Rev. X 1, 021019 (2011).

[33] Z. Li, S. Butun, and K. Aydin, Ultranarrow band absorbers based on surface lattice resonances in nanostructured metal surfaces, ACS Nano 8, 8242 (2014).

[34] X. Lu, R. Wan, and T. Zhang, Metal-dielectric-metal based narrow band absorber for sensing applications, Opt. Express 23, 29842 (2015).

[35] Y. Qu, Q. Li, H. Gong, K. Du, S. Bai, D. Zhao, H. Ye, and M. Qiu, Spatially and spectrally resolved narrowband optical absorber based on 2D grating nanostructures on metallic films, Adv. Opt. Mater. 4, 480 (2016).

[36] L. Meng, D. Zhao, Z. Ruan, Q. Li, Y. Yang, and M. Qiu, Optimized grating as an ultra-narrow band absorber or plasmonic sensor, Opt. Lett. 39, 1137 (2014).

[37] Y.-L. Liao and Y. Zhao, Graphene-based tunable ultranarrowband mid-infrared TE-polarization absorber, Opt. Express 25, 32080 (2017).

[38] B. Luk'yanchuk, N. I. Zheludev, S. A. Maier, N. J. Halas, P. Nordlander, H. Giessen, and C. T. Chong, The Fano resonance in plasmonic nanostructures and metamaterials, Nat. Mater. 9, 707 (2010).

[39] H. C. Hulst and H. C. van de Hulst, Light scattering by small particles (Courier Corporation, 1981).
[40] M. V. Rybin, K. B. Samusev, I. S. Sinev, G. Semouchkin, E. Semouchkina, Y. S. Kivshar, and M. F. Limonov, Mie scattering as a cascade of Fano resonances, Opt. Express 21, 30107 (2013).

[41] D. Rodrigo, O. Limaj, D. Janner, D. Etezadi, F. J. García de Abajo, V. Pruneri, and H. Altug, Mid-infrared plasmonic biosensing with graphene, Science 349, 165 (2015).

[42] N. Liu, M. L. Tang, M. Hentschel, H. Giessen, and A. P. Alivisatos, Nanoantenna-enhanced gas sensing in a single tailored nanofocus, Nat. Mater. 10, 631 (2011).

[43] P. B. Johnson and R.-W. Christy, Optical constants of the noble metals, Phys. Rev. B 6, 4370 (1972).

[44] A. Manjavacas, Anisotropic optical response of nanostructures with balanced gain and loss, ACS Photonics 3, 1301 (2016).

[45] W. Jin, C. Khandekar, A. Pick, A. G. Polimeridis, and A. W. Rodriguez, Amplified and directional spontaneous emission from arbitrary composite bodies: A self-consistent treatment of purcell effect below threshold, Phys. Rev. B 93, 125415 (2016).

[46] T. V. Teperik, F. J. García de Abajo, A. Borisov, M. Abdelsalam, P. Bartlett, Y. Sugawara, and J. Baumberg, Omnidirectional absorption in nanostructured metal surfaces, Nat. Photonics 2, 299 (2008).

[47] Z. Ruan and S. Fan, Superscattering of Light from Subwavelength Nanostructures, Phys. Rev. Lett. 105, 013901 (2010).

[48] COMSOL Multiphysics v. 5.3. www.comsol.com., COMSOL Inc, Stockholm, Sweden (2017).

[49] J. Yoon, K. H. Seol, S. H. Song, and R. Magnusson, Critical coupling in dissipative surface-plasmon resonators with multiple ports, Opt. Express 18, 25702 (2010).

[50] R. Stanley, Plasmonics in the mid-infrared, Nat. Photonics 6, 409 (2012).

[51] A. Grigorenko, M. Polini, and K. Novoselov, Graphene plasmonics, Nat. Photonics 6, 749 (2012).

[52] F. J. García de Abajo, Graphene plasmonics: Challenges and opportunities, ACS Photonics 1, 135 (2014).

[53] S. Thongrattanasiri, F. H. Koppens, and F. J. García de Abajo, Complete Optical Absorption in Periodically Patterned Graphene, Phys. Rev. Lett. 108, 047401 (2012).

[54] S. Kim, M. S. Jang, V. W. Brar, K. W. Mauser, L. Kim, and H. A. Atwater, Electronically tunable perfect absorption in graphene, Nano Lett. 18, 971 (2018).

[55] L. Zundel and A. Manjavacas, Spatially resolved optical sensing using graphene nanodisk arrays, ACS Photonics 4, 1831 (2017).

[56] A. I. Kuznetsov, A. E. Miroshnichenko, M. L. Brongersma, Y. S. Kivshar, and B. Luk'yanchuk, Optically resonant dielectric nanostructures, Science 354, aag2472 (2016).

[57] C. Wu, I. Burton Neuner, G. Shvets, J. John, A. Milder, B. Zollars, and S. Savoy, Large-area wide-angle spectrally selective plasmonic absorber, Phys. Rev. B 84, 075102 (2011).

[58] R. Alaee, M. Farhat, C. Rockstuhl, and F. Lederer, A perfect absorber made of a graphene micro-ribbon metamaterial, Opt. Express 20, 28017 (2012). 